\title{
Construction of Digital Culture Copyright Protection System Based on Blockchain Technology
}

\author{
Xiaoyun Zhou \\ Nanjing University of Posts and Telecommunications (NJUPT), Jiangsu, China
}

\begin{abstract}
Digital cultural copyright protection is an important means of digital cultural works protection. The existing digital cultural copyright protection has some problems in data storage security, rights confirmation efficiency, traceability means and copyright transaction. As an emerging technology in the Internet era, Blockchain can mobilize the enthusiasm of the whole network nodes and improve the security and reliability of digital culture copyright protection. By analyzing the applicability of Blockchain technology in digital culture copyright protection, a digital culture copyright protection system based on Blockchain technology is constructed.
\end{abstract}

Keywords: Blockchain, Digital culture copyright protection, League Chain.

\section{Introduction}

Digital cultural copyright protection is the protection of intellectual and technical property rights of digital cultural products spread through the network, including the digital copyright of literature, music, drama, painting, photography, pictures and films. Digital cultural copyright protection is the main means to protect the copyright of digital cultural works published on the Internet, and is used to control the use and dissemination of digital content (Gong Xue, 2018). In the new era, with the rapid development of all kinds of digital media and technology changes with each passing day, all kinds of new forms appear constantly, the public participation in the threshold gradually reduce, the cultural work to update and use frequency is accelerated, copyright protection of digital culture also put forward higher requirements.

\section{Analysis of Problems in Copyright Protection of Digital Culture}

Digital cultural copyright protection mainly includes two aspects: copyright confirmation and copyright utilization. Copyright confirmation is the confirmation of cultural copyright by the owner of digital cultural copyright. Copyright utilization is the trading process of digital cultural copyright works. It refers to the economic behavior of transferring or granting users the right to use copyright works and obtaining corresponding remuneration through copyright trading. At present, digital cultural works still have the following problems in data storage, copyright confirmation, copyright transaction and other aspects.

In terms of data storage, the existing digital culture copyright protection is generally stored through centralized database, with large storage capacity. In addition, when attacked, data is easy to be lost due to the lack of effective backup, which makes it difficult to guarantee the security and reliability of the system.

In the process of copyright confirmation, it is difficult to meet the demand of immediacy of digital cultural communication due to the tedious, time-consuming and inefficient process of copyright or patent application in special copyright management agencies; In the case of infringement, due to the lack of effective traceability means and the characteristics of digital works easy to tamper with and difficult to identify authenticity, it is difficult for copyright owners to provide proof and protect their rights, and they need to spend more energy and material resources to obtain evidence.

In the process of copyright trading, there are some problems such as hard matching between copyright owners and users, high intermediate cost and low efficiency, which are difficult to meet the demand of intelligent transaction in the digital era. There are information barriers between copyright owners and users, which cannot effectively communicate transaction demands; There are "channel intermediaries", such as distributors and music platforms in the film and television music industry. Centralized platforms make copyright owners in a weak position in the transaction and obtain a low share of market interests.

\section{The Applicability of Blockchain Technology in Digital Culture Copyright Protection}

Blockchain technology is a new application technology (Yuan Yong, Zhou Tao, Zhou Aoying, 2017). Through the application of Blockchain technology, it reduces the intermediate links of transactions, improves the participation enthusiasm of nodes in the whole network, and greatly improves the low efficiency and data storage security of traditional centralized institutions such as finance and logistics.

3.1 The Distributed Storage and Encryption Features of Blockchain Guarantee the Timeliness and Uniqueness of Copyright Grant

In the stage of copyright confirmation, the distributed database technology and time stamp technology of Blockchain can make each node in the Blockchain reach a quick consensus on the ownership of digital cultural copyright, ensuring the real-time process of copyright confirmation. At the same time, using the hash value and time stamp to prove that the existence, uniqueness and authenticity of digital content of a paragraph of text, audio of digital content, 
copyright owns can be convenient to complete copyright ownership certification.

\subsection{The Tamper-resistant and Traceable Characteristics of Blockchain Effectively Enhance the Strength of Copyright Protection of Digital Culture}

For copyright owners, the copyright and even creation process of works will be recorded on the chain through Blockchain technology, which will be endorsed by the digital cultural copyright management department to ensure the authenticity and reliability of data records. For copyright users, behavioral data such as registration, authentication, login, usage and transaction will be automatically recorded on the chain, and cannot be changed and destruction, realize the traceability of copyright trading process, which fundamentally solve the artificial evidence, evidence of long time, difficult problems.

\subsection{Consensus Mechanism Improves Trust and Engagement among Stakeholders}

The consensus mechanism improves the degree of trust and participation between subjects. Mutual trust between subjects on the chain is realized through the establishment of consensus mechanism. The subjects on the chain must pass the trusted authentication of each subject on the chain when registering, so as to ensure that the nodes added to the chain are trusted. Meanwhile, the subjects on the chain jointly maintain the operation of the Blockchain, realize the interconnection of information and the effective transfer of value.

\subsection{Smart Contract can Realize Automatic Transaction of Digital Cultural Copyright}

Using smart contract technology, digital culture copyright management department will take the lead in developing smart contracts that meet the interests of all subjects. When certain conditions are met, the smart contract will automatically execute the copyright transaction process and complete the value transfer of digital works. Meanwhile, smart contract can effectively avoid the intervention of middlemen, make the transaction process transparent, effectively solve the copyrighted content copy, distribution and profit channels, and create the largest economic income for copyright owners.

\section{Digital Culture Copyright Protection System based on Blockchain Technology}

\subsection{Blockchain Selection}

At present, Blockchain mainly includes public chain, private chain and alliance chain. For digital culture copyright protection, its main body mainly includes the copyright owners, users, and middlemen four groups, the administrative department and incomplete information made public, the body on the chain can obtain relevant access after the data, therefore, choose league chain technology system to achieve Blockchain in the application of digital culture copyright protection.

\subsection{Main Body on the Blockchain}

Digital culture copyright owner: the creator of digital culture copyright, which is generally an independent individual or team. The creator can $\log$ on the platform to confirm the copyright and manage the transaction of the work by himself or entrust a middleman for unified management.

Digital cultural copyright user: the entity that needs to purchase or use digital cultural copyright, which can be an individual or an organization, and needs to pay a certain fee for $t$ use or purchase of digital cultural copyright.

Digital culture copyright management department: it is the department responsible for digital culture copyright authorization and platform operation management. It is mainly responsible for the normal operation and maintenance of Blockchain, the establishment of consensus mechanism and smart contract, and the supervision of the behavior of the main body on the Blockchain.

Middleman: the agency responsible for representing the business of digital cultural copyright owners, charging a small amount of agency fees.

\subsection{Digital Culture Copyright Protection Architecture Based on Blockchain}

According to the actual application requirements of digital cultural copyright protection, the construction method of digital cultural copyright protection system based on Blockchain is proposed, as shown in Figure 1. From bottom to top, the first layer is the data layer, which encapsulates the block data chain structure and encryption mechanism supporting digital cultural copyright protection applications. The second layer is the network layer, which includes point-to-point communication networking and some security verification operations. The third layer is the consensus layer, which encapsulates the consensus mechanism of digital cultural copyright protection authenticated by each node to ensure that the process of right confirmation and transaction cannot be forged and denied. The fourth layer is the incentive layer, including the incentive mechanism for each subject in digital cultural copyright protection which participate in Blockchain operation and maintenance. The fifth layer is the contract layer, the contract layer is deployed to support the automatic transaction execution of digital cultural copyright intelligent contract, according to the transaction needs, the optimal match between copyright owners and users. 


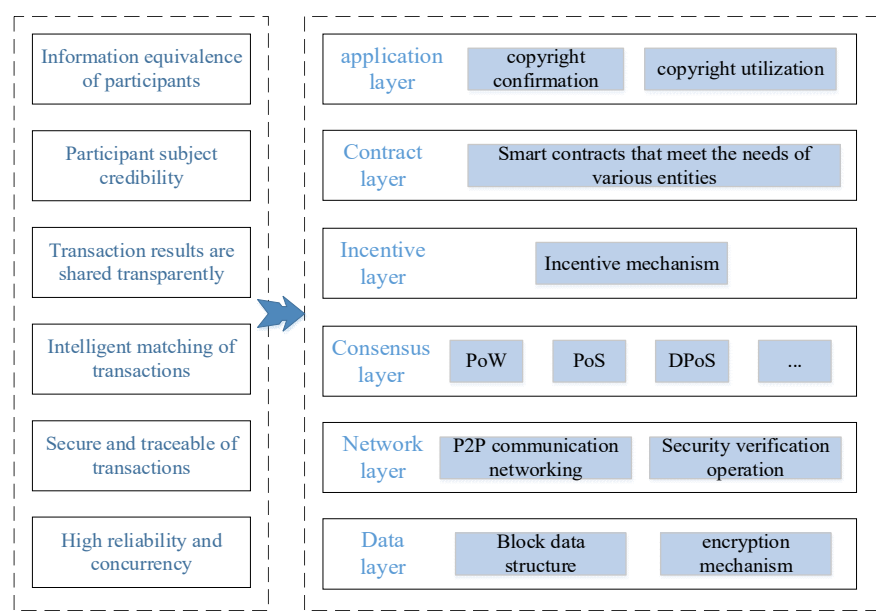

Figure 1: Digital culture copyright protection architecture based on Blockchain

\subsection{Digital Culture Copyright Protection Management Process Based on Blockchain}

According to the actual needs of digital culture copyright protection, copyrights confirmation and transaction process, the digital culture copyright protection management process based on Blockchain technology is shown in Figure 2.

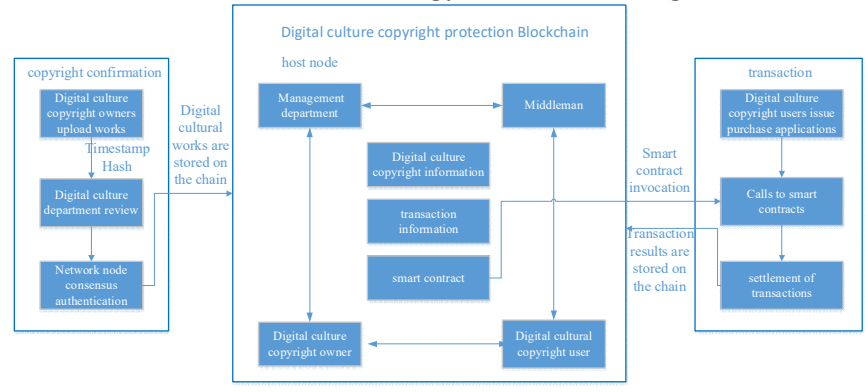

Figure 2: Digital culture copyright protection management process based on Blockchain

The first step is to establish Blockchain. Firstly, the main body of Blockchain is determined, including the owner, user, management department and middleman of digital cultural copyright. The management department is the main node, responsible for the establishment and operation of the chain. Secondly, the data stored on the chain should be clarified, including the confirmation data and transaction data. Finally, when the chain is completed, digital rights owners, users and middlemen are added to the chain as a node after being trusted.

The second step is for the digital culture copyright owner to complete the copyright certification. Firstly, the owner of digital cultural copyright logs into the system, uploads the cultural work to the chain, and generates a unique timestamp and hash value. Secondly, after the review of the management department, the whole network node carries on consensus authentication to the node uploaded to the chain; Finally, digital cultural works authenticated by consensus will be saved on the chain and jointly maintained by all network nodes, ensuring the reliability and security of digital cultural copyright protection.

The third step is the transaction process. Firstly, the digital culture copyright protection management department takes the lead in making the smart contract, which is signed and authenticated by each subject. The contract stipulates the responsibility of the subject of the transaction, the process of the transaction, the confirmation method of the transaction amount, the transaction settlement mode, the scope of copyright use and other contents. Secondly, when the copyright user needs to use or purchase copyright, it will automatically identify the transaction type, execute the transaction automatically according to the terms of the smart contract, and complete the payment. Finally, the transaction process information will be recorded on the chain, and the main body of the chain has the authority to open.

\section{Conclusions}

Blockchain technology application in the field of digital culture copyright protection will promote the involvement of different parts, realize the digital culture copyright protection transforms from a centralized line management to distributed online, will effectively promote the deepening of the age of the Internet culture industry transformation, the greater the utilization level of enhance the value of copyright owners and their work.

\section{References}

[1] Gong Xue. Application prospect analysis of Blockchain digital copyright protection technology [J]. Communication \& Copyright, 2018(7): 182-184.

[2] Yuan Yong, Zhou Tao, Zhou Aoying, et al. Blockchain technology: from data intelligence to knowledge automation [J]. Automatica, 2017, 43(9): 1485-1490.

\section{Author Profile}

Xiaoyun Zhou, Senior Researcher, Nanjing University of Posts and Telecommunications (NJUPT), Jiangsu, China. 